\title{
The role of Hashimoto thyroiditis in predicting radioiodine ablation efficacy and prognosis of low to intermediate risk differentiated thyroid cancer
}

\author{
Domenico Albano ${ }^{1}$ (D) - Francesco Dondi ${ }^{1}$. Valentina Zilioli ${ }^{2} \cdot$ Maria Beatrice Panarotto $^{2} \cdot$ Alessandro Galani $^{3}$. \\ Carlo Cappelli ${ }^{4} \cdot$ Francesco Bertagna $^{1} \cdot$ Raffaele Giubbini $^{1} \cdot$ Claudio Casella $^{5}$
}

Received: 17 March 2021 / Accepted: 13 June 2021 / Published online: 21 June 2021

(c) The Author(s) 2021

\begin{abstract}
Objective The baseline treatment of differentiated thyroid cancer (DTC) consists of thyroidectomy followed by postoperative risk-adapted radioiodine therapy (RAIT) when indicated. The choice of most appropriate RAI activities to administer with the aim to reach an efficient remnant ablation and reduce the risk of recurrence is yet an open issue and the detection of basal factors that may predict treatment response seems fundamental. The aim of this study was to investigate the potential role of Hashimoto thyroiditis (HT) in predicting 1-year and 5-year treatment response after RAIT and prognosis.

Methods We retrospectively included 314 consecutive patients ( 174 low-risk and 140 intermediate-risk) who received thyroidectomy plus RAIT. One-year and 5-year disease status was evaluated according to 2015 ATA categories response based upon biochemical and structural findings.

Results HT was reported histopathologically in 120 patients (38\%). DTC patients with concomitant HT received a higher number of RAITs and cumulative RAI activities. Initial RAIT reached an excellent response in $63 \%$ after one year and $84 \%$ after 5 years. The rate of excellent response one year and 5-year after first RAIT was significantly lower in HT groups, compared to not HT $(p<0.001)$. Instead, HT did not have a prognostic role considering PFS and OS; while stimulate thyroglobulin (sTg) at ablation was significantly related to survival.

Conclusions HT may affect the efficacy of RAIT in low to intermediate risk DTC, particularly reducing the successful rate of excellent response after RAIT. Instead, HT did not have a prognostic impact such as stimulated sTg.
\end{abstract}

Keywords RAI · Differentiated thyroid cancer $\cdot$ Hashimoto thyroiditis $\cdot$ Ablation

Domenico Albano

doalba87@libero.it

1 Nuclear Medicine, University of Brescia and ASST Spedali Civili Brescia, Brescia, Italy

2 Nuclear Medicine Department, ASST Spedali Civili Brescia, Brescia, Italy

3 Department of Clinical and Experimental Sciences, Surgical Clinic, University of Brescia, Brescia, Italy

4 Department of Clinical and Experimental Sciences, SSd Medicina ad Indirizzo Endocrino-Metabolico, University of Brescia, Brescia, Italy

5 Department of Molecular and Translation Medicine, Surgical Clinic, University of Brescia, Brescia, Italy

\section{Introduction}

Differentiated thyroid cancer (DTC) is the most common endocrine malignancy, accounting for $1 \%$ of all cancers diagnosed each year [1,2] and it is considered a slowly growing cancer with an overall good outcome, except of cases with distant metastases or radioiodine-refractory disease [3, 4]. The baseline treatment of DTC consists of thyroidectomy followed by postoperative risk-adapted radioiodine therapy (RAIT) when indicated $[2,5]$.

The choice of most appropriate RAI activities to administer with the aim to reach an efficient remnant ablation and reduce the risk of recurrence is yet an open issue. This point is even more debated in low-risk and intermediate-risk DTC, where it seems crucial to find the best balance between the effective cure and the risk of side effects (reduced radiation exposure, cost, time of hospitalization) [6-9]. Consensus 
guidelines recommend a dose of 1.1-3.7 GBq for patients with low to intermediate-risk DTC and higher activities for high-risk DTC, residual disease or aggressive histological subtypes [2]. Several papers [10, 11] demonstrated the equivalence of successful ablation between low-activity $(1.1 \mathrm{GBq})$ and high-activity $(3.7 \mathrm{Gbq})$ and between thyroid hormone withdrawal and recombinant human thyroid-stimulating hormone injections, without significant difference in outcome survival.

To better understand which activity inject in these patients successfully while minimizing the side effects, it seems fundamental to know the factors that influence the success of ablation and to predict the outcome.

The most shared known factors include the preablation stimulated thyroglobulin (sTg) level, the completeness of surgery, the preablation radioiodine scan findings and the histotype [12-14]. The potential influence of Hashimoto Thyroiditis (HT) in the effectiveness of RAIT is not wellinvestigated. Only Kwon et al. [15] showed that coexisting HT together with sTg were negative predictive factors for successful low-dose RAIT (1.1 GBq).

Moreover, also in predicting treatment response and prognosis the role of HT is not well-investigated. The association between DTC and HT has been long suggested with several studies in favor [16-18]. Probably, HT is frequently associate with DTC, especially papillary thyroid carcinoma, and may be considered a risk factor for developing thyroid cancer but the reason of this link is not yet understood [19, 20]. Moreover, DTC patients with concomitant HT seems to have a better prognosis that DTC patients without [21, 22].

The main aim of this study was to retrospectively investigate in a consecutive cohort of patients with low- to intermediate-risk DTC treated with total thyroidectomy and RAIT, the role of HT in predicting 1-year and 5-year treatment response and disease status. The second aim was to analyze the potential prognostic role of HT in this population.

\section{Materials and methods}

We retrospectively screened all patients who performed thyroidectomy from January 2010 until January 2018 in our Surgery Department. Inclusion criteria were (1) a total thyroidectomy followed by RAIT, (2) age more or equal to 18 years at time of surgery, (3) a histological confirmation of DTC, (4) a classification as low risk or intermediate risk according to 2015 ATA guidelines [2], (5) at least 12 months of clinical and imaging follow-up after RAIT. Exclusion criteria were (1) age less than 18-years old, (2) hypothyroidism or hyperthyroidism condition before surgery, (3) a previous history of other neoplastic disease. Finally, 314 patients were included: 174 low-risk class stage after surgery and RAIT and 140 intermediate-risk class. There was a prevalence of female ( $\mathrm{F}: \mathrm{M}=2.8: 1)$ and mean age at diagnosis was 50-years old (Table 1). The first administered activity of RAI ablation ranged from 1.1 to $4.4 \mathrm{GBq}$ (average $2.7 \mathrm{GBq}$ ) and it was established according to the risk class based on the TNM staging of the AJCC currently in use. Patients treated before the introduction of new American Joint Committee on Cancer/International Union against Cancer (AJCC) 8th edition were reclassified according to the last guideline [23]; this explains why some patients were treated with high-activities due to the downstage from intermediate-risk class to lowrisk one.

All patients underwent total thyroidectomy and had a histopathological diagnosis of DTC: 146 classic variant of papillary carcinoma, 105 follicular variant of papillary carcinoma, 25 follicular carcinoma (17 minimally invasive and 8 widely invasive), 26 aggressive papillary variants (18 tall cells variant of papillary carcinoma and 8 sclerosing diffuse variant of papillary carcinoma) and 12 Hürthle cell carcinoma. One hundred and fifty patients underwent also a central lymphadenectomy and also lateral in fifty-four cases. HT was reported at the histological sample in 120 patients (38\%), while in the remaining 194 cases $(62 \%)$ no foci of HT were described. All patients received RAIT 2-3 months after thyroidectomy: 137 patients discontinued levothyroxine treatment 4 weeks before radioactive-iodine remnant ablation and replaced by triiodothyronine for 2 weeks, while 177 patients received recombinant human thyrotropin (rhTSHThyrogen, Genzyme Corporation) intramuscularly with a dose of $0.9 \mathrm{mg}$ over 2 consecutive days during treatment with levothyroxine and RAI was administered the day after the second injection. Serum thyrotropin (TSH), fT3, fT4, Tg, antithyroglobulin antibodies $(\mathrm{TgAb})$, and ioduria were measured before RAIT. All patients followed a low-iodine diet for at least 2 weeks and mean TSH level was higher than 30 $\mathrm{UI} / \mathrm{L}$ in all cases. Three days after RAIT, the patients underwent a whole body scintigraphy (WBS) followed by single photon emission computed tomography/computed tomography (SPECT/CT), by hybrid dual-detector SPECT/CT (Infinia Hawkeye II, GE Healthcare, Haifa Israel), equipped with 1 inch StarBrite ${ }^{\mathrm{TM}}$ Crystal and a high-energy collimator. The StarBrite ${ }^{\mathrm{TM}}$ Crystal has a sensitivity more than twofolds higher than that provided by a $3 / 8$ inch crystal without a significant loss in resolution, therefore contributing to improve the reliability of SPECT with RAI. The WBS was performed in continuous mode with high-energy general purpose (HEGP) parallel holes collimator, 364-keV photopeak with $\pm 10 \%$ energy windows setting and scatter correction. The infrared-based real-time automatic body contouring system was activated for simultaneous anterior and posterior view with a matrix of $256 \times 1024$. Hybrid SPECT/CT scans from skull base to the lung bases were routinely acquired in all cases and additional SPECT/CT scans of other areas were done according to the WBS findings. SPECT images were 
Table 1 Baseline features of our population

\begin{tabular}{|c|c|c|}
\hline & Average \pm SD (range) & Patients $n(\%)$ \\
\hline Age years & $50 \pm 14(18-85)$ & \\
\hline \multicolumn{3}{|l|}{ Gender } \\
\hline Male & & $82(26 \%)$ \\
\hline Female & & $232(74 \%)$ \\
\hline \multicolumn{3}{|l|}{ Histotype } \\
\hline Papillary & & $146(46 \%)$ \\
\hline Follicular variant of papillary & & $105(34 \%)$ \\
\hline Follicular & & $25(8 \%)$ \\
\hline Aggressive variant (tall cells and sclerosing diffuse) & & $26(8 \%)$ \\
\hline Hurtle cell & & $12(4 \%)$ \\
\hline Lymphatic invasion & & $91(29 \%)$ \\
\hline Vascular invasion & & $76(24 \%)$ \\
\hline Extrathyroidal extension & & $110(35 \%)$ \\
\hline Resection margin involvement & & $49(16 \%)$ \\
\hline Tumor size (mm) & $18.4 \pm 15.2(1-85)$ & \\
\hline Multicentricity & & $160(51 \%)$ \\
\hline Hashimoto thyroiditis & & $120(38 \%)$ \\
\hline \multicolumn{3}{|l|}{ T-stage } \\
\hline sT1 & & $179(62 \%)$ \\
\hline sT2 & & $47(10 \%)$ \\
\hline sT3 & & $88(28 \%)$ \\
\hline \multicolumn{3}{|l|}{ N-stage } \\
\hline sN0 & & $240(78 \%)$ \\
\hline sN1a & & $30(9 \%)$ \\
\hline sN1b & & $44(14 \%)$ \\
\hline \multicolumn{3}{|l|}{ ATA class risk } \\
\hline Low & & $174(55 \%)$ \\
\hline Intermediate & & $140(45 \%)$ \\
\hline Pre-ablation $\mathrm{Tg}$ & $1.8 \pm 2.2(0.1-100)$ & \\
\hline $\mathrm{sTg}$ at the time of ablation $(\mathrm{ng} / \mathrm{mL})$ & $10.5 \pm 25.9(0.1-250)$ & \\
\hline $\operatorname{TgAb}$ positive at ablation & & $114(36 \%)$ \\
\hline First RAI activities administrated (GBq) & $2.7 \pm 1.2(1-4.4)$ & \\
\hline Cumulative RAI activities administrated (GBq) & $6.3 \pm 8(1-56)$ & \\
\hline No. therapies & $1.5 \pm 1(1-7)$ & \\
\hline \multicolumn{3}{|l|}{ 1-year treatment-response categories } \\
\hline Excellent response & & $199(63 \%)$ \\
\hline Indeterminate response & & $53(17 \%)$ \\
\hline Biochemical and/or structural incomplete response & & $62(20 \%)$ \\
\hline \multicolumn{3}{|l|}{ 5-year treatment-response categories } \\
\hline Excellent response & & $264(84 \%)$ \\
\hline Indeterminate response & & $12(4 \%)$ \\
\hline Biochemical and/or structural incomplete response & & $19(6 \%)$ \\
\hline Progression & & $4(1 \%)$ \\
\hline $\mathrm{Na}$ & & $15(5 \%)$ \\
\hline
\end{tabular}

$n$ number, $G B q$ Gigabequerel, $R A I$ radioiodine, var variant, $s T g$ stimulated thyroglobulin, $A b$ antibodies, $n a$ not available acquired with HEGP collimator, matrix size of $128 \times 128$, 364-keV photopeak with $\pm 10 \%$ energy and scatter windows, dual-detector $180^{\circ}$ acquisition, angular step of $3^{\circ}, 15^{\prime \prime}$ time per step/view. The CT parameters were $140 \mathrm{kV}, 2.5 \mathrm{~mA}$, 30 " rotation speed, $10 \mathrm{~mm}$ slice thickness, $256 \times 256$ matrix. $\mathrm{CT}$ acquisition was performed with a 2 -slices elicoidal 
acquisition. An ordered subset expectation maximization iterative reconstruction with CT-based attenuation correction and scatter correction was performed. Moreover, about one-year after RAIT, a WBS after a diagnostic activity of $185 \mathrm{MBq}$ or after a therapeutic activity in case of persistent disease was executed. Also sTg, TSH and TgAb were done at the same time and the combination of biochemical and radiological findings were used to classify the ablation success rate and the treatment response categories according to ATA groups (excellent response, biochemical incomplete response, structural response and indeterminate response) [2]. Disease status 5 year after RAIT was evaluated considering a combination of clinical, biochemical ( $\mathrm{Tg}, \mathrm{AbTg})$ and imaging (ultrasound, CT, WBS and/or PET/CT) data.

\section{Statistical analysis}

All statistical analyses were carried out using Statistical Package for Social Science (SPSS) version 23.0 for Windows (IBM, Chicago, Illinois, USA) and MedCalc Software version 17.1 for Windows (Ostend, Belgium). The descriptive analysis of categorical variables comprised the calculation of simple and relative frequencies; the numeric variables were described as mean, standard deviation, minimum and maximum.

The statistical significance of the categorical variables was tested with $\chi^{2}$ or Fisher's exact test and a Student's $t$-test or Mann-Whitney's $U$-test were performed for the continuous features. A $p$ value $<0.05$ was considered statistically significant.

Progression-free survival (PFS) was calculated from the date of diagnosis to the date of first disease progression, relapse, death or the date of last follow-up. Overall survival (OS) was calculated from the date of diagnosis to the date of death from any cause or to the date of last follow-up. Survival curves were plotted according to the Kaplan-Meier method and differences between groups were analyzed using a two-tailed log rank test. Cox regression was used to estimate the hazard ratio (HR) and its confidence interval (CI). A $p$ value $<0.05$ was considered statistically significant.

\section{Results}

\section{Treatment response evaluation}

Patients were divided in two groups according to the presence of HT: group 1 consists of $194(62 \%)$ patients without HT and group 2 of 120 (38\%) with HT concomitant to DTC (Table 2).

Considering the main epidemiological (age, gender), histological (histotype, lymphatic invasion, vascular invasion, extrathyroidal invasion, resection margin involvement, multicentricity and tumor size) and clinical features (ATA class risk, nodal disease at staging, pre-ablation $\mathrm{Tg}$ and sTg) not significant differences were present between the two groups. On the other hand, the presence of AbTg was significantly higher in HT groups than non HT ( $57 \%$ vs $24 \%$, $p<0.001$ ).

Also cumulative RAI activities administrated and number of radiometabolic therapies were higher in patients with coexisting HT than those without HT ( $p<0.001$ for both).

Most of patients (63\%) had an excellent response one year after RAIT, $17 \%$ an indeterminate response, $20 \%$ an incomplete response ( $5 \%$ only biochemical; $2 \%$ only structural and $13 \%$ both biochemical and structural) (Table 1). Patients with coexisting HT showed a lower rate of 1-year excellent response (54\%) than without HT $(69 \%)(p<0.001)$ (Fig. 1a). In patients without HT, 1-year not excellent response consisted of 21 cases of indeterminate response and 39 of incomplete response (27 both biochemical and structural; 7 only biochemical and 5 only structural). While, in patients with HT, 1-year not excellent response consisted of 31 cases of indeterminate response and 24 of incomplete response (20 both biochemical and structural; 3 only biochemical and 1 only structural) (Fig. 1b).

Comparing neck ultrasound findings 6-12 months after first radiometabolic therapy, no differences were described between the two groups (HT vs no HT).

Considering disease status five year after RAI first therapy, the rate of excellent response was $84 \%$, of indeterminate response $4 \%$, incomplete response $6 \%$ and progression $1 \%$. In 15 patients the 5-year disease status was not available.

Patients with coexisting HT showed a lower rate of 5-year excellent response (79\%) than without HT (94\%) $(p<0.001)$ (Fig. 2a). In patients without HT, 5-year not excellent response consisted of 3 cases of indeterminate response, 7 of incomplete response ( 6 both biochemical and structural and 1only biochemical) and one of progression. Instead in patients with HT, 5-year not excellent response consisted of 10 cases of indeterminate response, 10 of incomplete response ( 7 both biochemical and structural; 2 only biochemical and 1 only structural) and 4 of progression (Fig. 2b).

\section{Prognosis}

At a median follow-up of 88 months, relapse or progression of disease occurred in 23 patients with an average time of 60.8 months (8-204 months), while death occurred in 5 patients with an average time of 78.4 months (55-151). The median PFS was 85 months (8-300 months) and the median OS was 88 months (10-300 months). In univariate analysis, gender, age, ATA class risk group, sTg and disease status after 1 and 5 years are significantly related with PFS (Table 3 ). 
Table 2 comparison between DTC patients with concomitant HT and without

\begin{tabular}{|c|c|c|c|}
\hline Variable & no HT $n 194$ & HT $n 120$ & $p$ value \\
\hline Age, mean (years) & 51.3 & 48.2 & 0.058 \\
\hline Gender F:M & $139: 55$ & $93: 27$ & 0.252 \\
\hline Histotype & & & 0.090 \\
\hline Papillary & $86(44 \%)$ & $60(50 \%)$ & \\
\hline Follicular variant of papillary & $65(33 \%)$ & $40(33 \%)$ & \\
\hline Follicular & $22(11 \%)$ & $3(3 \%)$ & \\
\hline Aggressive variant & $13(7 \%)$ & $13(11 \%)$ & \\
\hline Hurtle cell & $8(4 \%)$ & $4(3 \%)$ & \\
\hline Lymphatic invasion & $54(28 \%)$ & $37(31 \%)$ & 0.570 \\
\hline Vascular invasion & $46(24 \%)$ & $30(25 \%)$ & 0.796 \\
\hline Extrathyroidal extension & $67(35 \%)$ & $43(36 \%)$ & 0.891 \\
\hline Resection margin involvement & $33(17 \%)$ & $16(13 \%)$ & 0.385 \\
\hline Tumor size (mm) & 18.3 & 18.8 & 0.747 \\
\hline Multicentricity & $95(49 \%)$ & $65(54 \%)$ & 0.372 \\
\hline ATA class risk & & & 0.295 \\
\hline Low & $112(58 \%)$ & $62(51 \%)$ & \\
\hline Intermediate & $82(42 \%)$ & $58(49 \%)$ & \\
\hline Lymph node metastasis & $41(21 \%)$ & $34(28 \%)$ & 0.289 \\
\hline Pre-ablation Tg (ng/mL) & 1.9 & 1.8 & 0.887 \\
\hline $\mathrm{sTg}$ at the time of ablation $(\mathrm{ng} / \mathrm{mL})$ & 8.4 & 7.5 & 0.470 \\
\hline $\mathrm{TgAb}$ positive at ablation & $46(24 \%)$ & $68(57 \%)$ & $<0.001$ \\
\hline First RAI activities administrated $(\mathrm{GBq})$ & 2.6 & 2.8 & 0.242 \\
\hline Cumulative RAI activities administrated (GBq) & 5.1 & 8.2 & $<0.001$ \\
\hline $\mathrm{N}^{\circ}$ therapies & 1.3 & 1.7 & 0.001 \\
\hline 1-year treatment-response categories & & & $<0.001$ \\
\hline Excellent response & $134(69 \%)$ & $65(54 \%)$ & \\
\hline Not Excellent response & $60(31 \%)$ & $55(46 \%)$ & \\
\hline 5-year treatment-response categories & & & $<0.001$ \\
\hline Excellent response & $171(94 \%)$ & $93(79 \%)$ & \\
\hline Not Excellent response & $11(6 \%)$ & $24(21 \%)$ & \\
\hline Data non available & 12 & 3 & \\
\hline Recurrence & $11(6 \%)$ & $12(10 \%)$ & 0.153 \\
\hline Death & $3(2 \%)$ & $2(2 \%)$ & 0.934 \\
\hline
\end{tabular}

Stimulated Tg was dichotomized using ROC curve analysis and $3.8 \mathrm{ng} / \mathrm{mL}$ was derived as best threshold (AUC 0.775 , sensitivity $100 \%$, specificity $71 \%, p<0.001)$. The other clinical and pathological features, like HT, were not significantly associated with PFS. In multivariate analysis, age, sTg, 1-year disease status and 5-year disease status were confirmed to be independent prognostic factors for PFS (Fig. 3).

While considering OS, at univariate analysis age, sTg, cumulative RAI activities administered and total number of RAIT were significantly correlated with death. At multivariate analysis, only sTg was confirmed to be an independent prognostic variable (Table 3; Fig. 3).

\section{Discussion}

HT is the most common autoimmune inflammatory thyroid disease with a worldwide annual incidence of $0.3-1.5$ cases for 1000 persons and it is considered the first cause of hypothyroidism in the iodine-sufficient areas of the world [24]. The etiopathogenesis is probably a combination between environmental factors and genetic condition which causes the loss of immunological tolerance, with a subsequent autoimmune attack to the thyroid tissue. HT brings a chronic inflammatory condition that awakens an immune response leading to a continuous damage 
Fig. 1 Histogram distribution of treatment response categories one year after first RAIT a
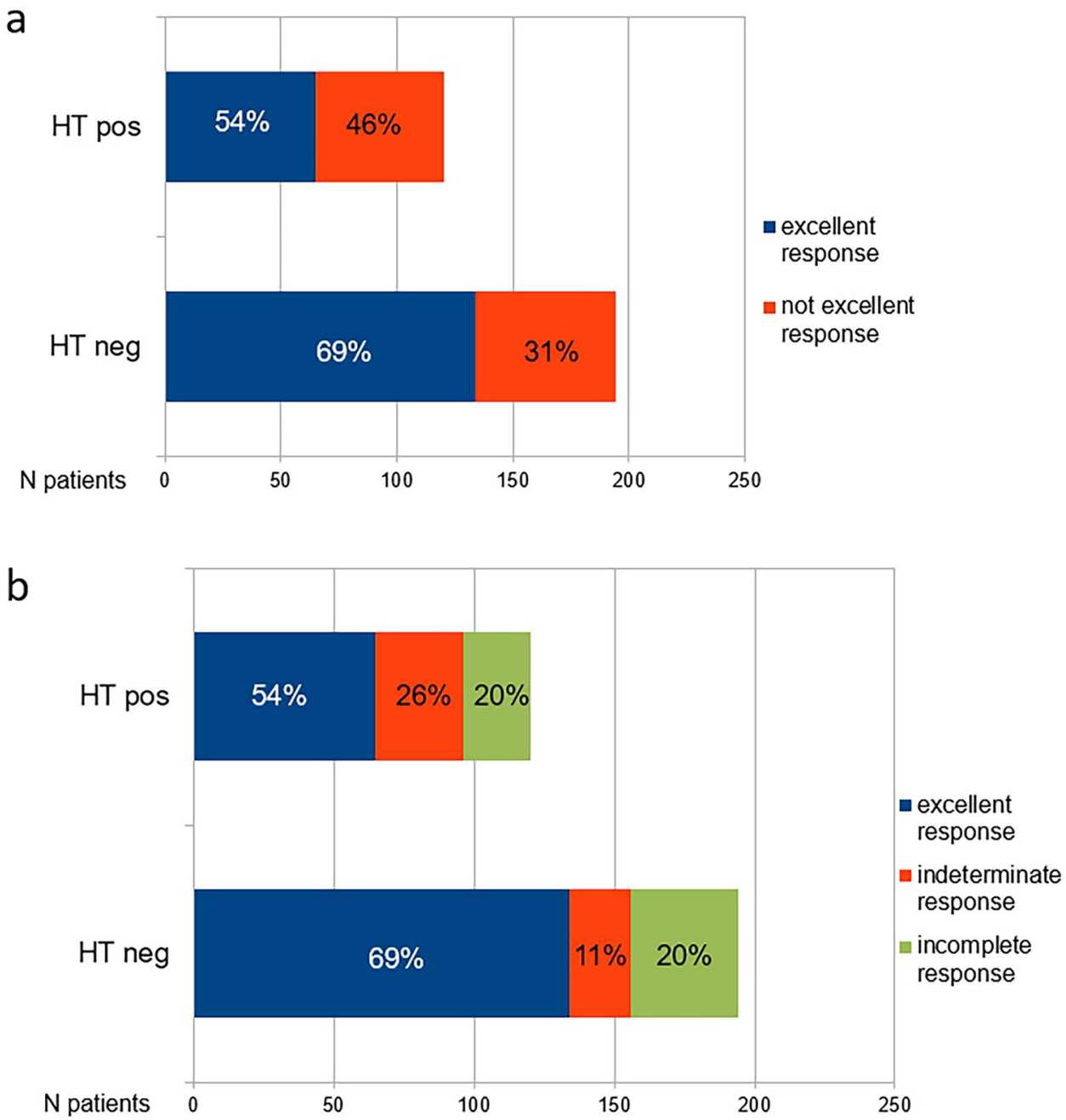

of surrounding stromal cells; overall this phenomenon causes potential genetic alterations, an inappropriate cell proliferation, and increases the risk of neoplastic transformation. Historically, HT was demonstrated significantly associate with an increased risk of developing thyroid lymphoma [25, 26]. But also the association between HT and DTC has been investigated with positive evidences [19, 20]. First in 1955 Dailey et al. [27] reported an increased association between HT and DTC, mainly papillary TC. However, the reason of this link remains yet unclear. The incidence of HT with concomitant PTC were reported as $26.8-38.1 \%$ in Italy, and $14.7-30.1 \%$ in the United States [28]. There are present in literature several hypotheses: someone suggested that HT could be a "cancer-impending effect" meaning an attempt of autoimmune system to fight against the tumor with the aim to cure the oncological disease and it could explain the favorable outcome reported in DTC with concomitant HT [29]; others speculated that the elevated TSH levels, often secondary to HT, might stimulate follicular epithelial cells proliferation and gained also the proliferation of cancer cells [30], but no shared consensus is available.

In our sample, the two groups (HT vs. no HT) had similar epidemiological and histological features, despite the histotype variants distribution and the age were near to statistical significance ( 0.090 for histotype and 0.058 for age). Patients with HT had lower age at diagnosis and less cases of follicular DTC in comparison with no HT patients.

Beside the linkage between HT and DTC, it seems crucial to clarify if conventional therapy of DTC may be affected by the presence or not of HT. Kwon et al. [15] conducted a retrospective analysis on 691 DTC patients who underwent thyroidectomy plus $1.1 \mathrm{GBq}$ of RAI. Successful RAI ablation was registered in $62.3 \%$ of patients, and stimulated $\mathrm{Tg}$ at ablation and coexisting HT were the only two factors significantly associated with the success rate. The efficacy of lowactivity of RAI was negatively influenced by sTg and HT. Moreover, patients with HT underwent more RAI therapies and higher total RAI activity. These evidences were confirmed also by our analysis: in DTC patients with coexisting 
Fig. 2 Histogram distribution of treatment response categories 5 years after first RAIT

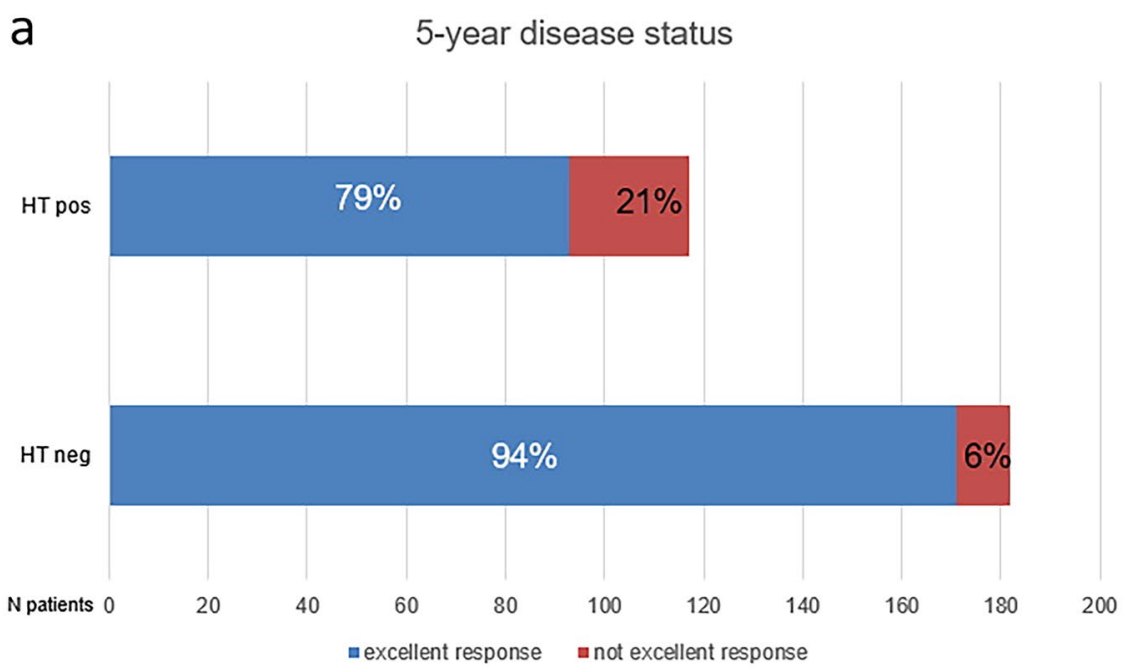

b 5-year disease status

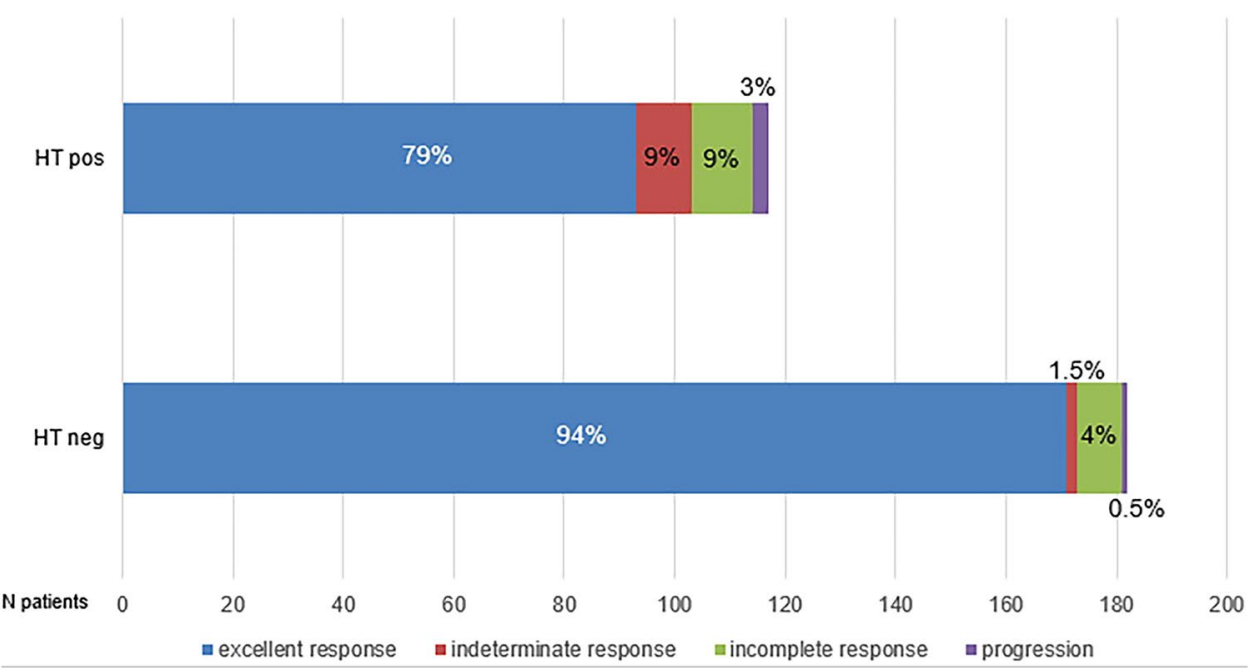

HT the rate of 1-year excellent response was lower than DTC patients without HT (54\% vs $69 \% ; p<0.001)$ and also the 5-year excellent response was lower than DTC patients without HT ( $79 \%$ vs $94 \%$; $p<0.001)$. One possible explanation for RAI failure is the reduction of lower sodium-iodide symporter (NIS) expression in patients with coexisting HT, which it is strictly related to the decreased radioactive iodine uptake into the remnant thyroid tissue [31]. Kollecher et al. [32] demonstrated that thyroid tissue with HT showed significantly lower levels of NIS membrane staining than normal thyroid tissue. Moreover, the inflammatory background typical of HT, may reduce the therapeutic efficacy of RAI. Also for high-activity (3.7 GBq) HT seems to have a significant role in predicting ablation success after RAI [33]: in patients with HT the success rate was $33.4 \%$ in comparison with DTC without HT with a rate of $66.6 \%$. In our study, we included both low and intermediate risk class DTC, thus low-intermediate RAI activity was injected. The presence of HT was related with higher cumulative RAI activities administrated and higher number of radiometabolic therapies; also in this case, the reason could be related with the less efficacy of radioiodine to enter in the thyroid cells due to the inflammatory micro-environment and the loss NIS expression.

In the last 2015 ATA guidelines [2], for the first time a new category response was introduce called indeterminate response which consist of patients without clear biochemical or structural disease, but without a complete response. In this group it is included also the case of DTC patient with $\mathrm{TgAb}$ stable or declining, but positive, without definitive structural evidence of disease. Two studies have demonstrated that only $13-20 \%$ of patients with an indeterminate response to therapy are reclassified as persistent/recurrent disease over approximately 10 years of follow-up [34, 35]

The rate of patients with indeterminate response after RAIT was significantly higher in HT patients compared to 
Table 3 Univariate and multivariate analyses for PFS and OS

\begin{tabular}{|c|c|c|c|c|}
\hline & \multicolumn{2}{|c|}{ Univariate analysis } & \multicolumn{2}{|c|}{ Multivariate analysis } \\
\hline & $p$ value & HR $(95 \% \mathrm{CI})$ & $p$ value & $\mathrm{HR}(95 \% \mathrm{CI})$ \\
\hline \multicolumn{5}{|l|}{ PFS } \\
\hline Gender & 0.046 & $0.398(0.160-0.987)$ & 0.251 & $2.222(0.859-4.520)$ \\
\hline Age $(<55$ years vs $\geq 55)$ & 0.004 & $0.282(0.117-0.683)$ & 0.004 & $3.590(1.482-8.691)$ \\
\hline ATA class risk (low vs intermediate) & 0.023 & $0.274(0.119-0.629)$ & 0.281 & $2.945(0.345-7.728)$ \\
\hline Histotype & 0.210 & $2.123(0.872-5.449)$ & & \\
\hline HT & 0.250 & $0.610(0.269-1.416)$ & & \\
\hline Multifocality & 0.584 & $0.772(0.332-1.794)$ & & \\
\hline Vascular invasion & 0.600 & $0.998(0.235-2.987)$ & & \\
\hline Lymphatic invasion & 0.251 & $0.567(0.215-1.495)$ & & \\
\hline Tumor size & 0.187 & $0.443(0.100-1.435)$ & & \\
\hline $\operatorname{TgAb}$ presence & 0.298 & $1.580(0.666-3.748)$ & & \\
\hline $\mathrm{sTg}$ at ablation (cutoff $3.8 \mathrm{ng} / \mathrm{mL}$ ) & 0.004 & $0.278(0.116-0.668)$ & 0.027 & $2.748(1.151-6.560)$ \\
\hline 1-year disease status* & 0.003 & $0.201(0.085-0.475)$ & 0.009 & $1.540(1.222-1.879)$ \\
\hline 5 year disease status* & 0.001 & $0.076(0.021-0.273)$ & 0.010 & $2.435(1.200-5.987)$ \\
\hline \multicolumn{5}{|l|}{ OS } \\
\hline Gender & 0.304 & $0.252(0.041-1.531)$ & & \\
\hline Age & 0.040 & $0.148(0.002-0.922)$ & 0.198 & $1.876(0.546-3.454)$ \\
\hline ATA class risk & 0.134 & $0.252(0.041-1.531)$ & & \\
\hline Histotype & 0.657 & $1.694(0.235-12.398)$ & & \\
\hline HT & 0.895 & $1.127(0.183-6.826)$ & & \\
\hline Multifocality & 0.879 & $1.151(0.186-7.125)$ & & \\
\hline Vascular invasion & 0.090 & $0.183(0.0241 .335)$ & & \\
\hline Lymphatic invasion & 0.078 & $0.163(0.021-1.227)$ & & \\
\hline Tumor size & 0.224 & $0.198(0.098-1.987)$ & & \\
\hline $\mathrm{TgAb}$ presence & 0.070 & na & & \\
\hline sTg at ablation & 0.002 & na & 0.034 & $1.890(1.121-2.876)$ \\
\hline 1-year disease status* & 0.345 & $0.415(0.065-2.596)$ & & \\
\hline 5 year disease status* & 0.545 & $0.418(0.025-6.937)$ & & \\
\hline Total no. RAITs & 0.039 & na & 0.212 & $1.333(0.879-2.001)$ \\
\hline Cumulative RAI activities administrated (GBq) & 0.011 & na & 0.234 & $1.010(0.768-2.221)$ \\
\hline
\end{tabular}

PFS progression-free survival, $O S$ overall survival, $H R$ hazard ratio, $C I$ confidence interval

*Variables dichotomized as excellent response vs not excellent response

not HT both 1 year and 5 years after RAIT. This is directly associate with the presence of $\operatorname{TgAb}$ in patients with HT compared in patients without. The high rate of indeterminate response could partially explain the different excellent response rate between the two groups, even though the prognosis is not affected.

On the other hand, HT does not show a prognostic role in predicting PFS and OS. Other parameters, like age at diagnosis, sTg, and disease status after first-line therapy were significantly correlated with the risk of recurrence. Stimulated Tg confirmed to be an independent prognostic variable also for OS. Several papers underlined a possible association between HT and a good outcome, describing that DTC with concomitant HT had a lower rate of lymph node and distant metastases [22, 36, 37]. In our analysis, no significant impact of HT in prognosis was showed also due to the selection of low- to intermediate-risk DTC, which for definition have a good prognosis. In fact, the number of events (progression/relapse or death) was relatively low. As previously demonstrated [2], preablation sTg level has been considered a poor prognostic factor for successful ablation and prognosis. In this manuscript, a sTg threshold of $3.8 \mathrm{ng} / \mathrm{mL}$ was derived as the best compromise between sensitivity and specificity; other cutoff values are suggested in literature [38, 39].

Our study had several limitations, including the retrospective nature of the study design, the heterogeneity of patients' features and the relatively not long follow-up time (88 months in this study) for prognostic evaluation considering the outcome of low to intermediate risk DTC. 

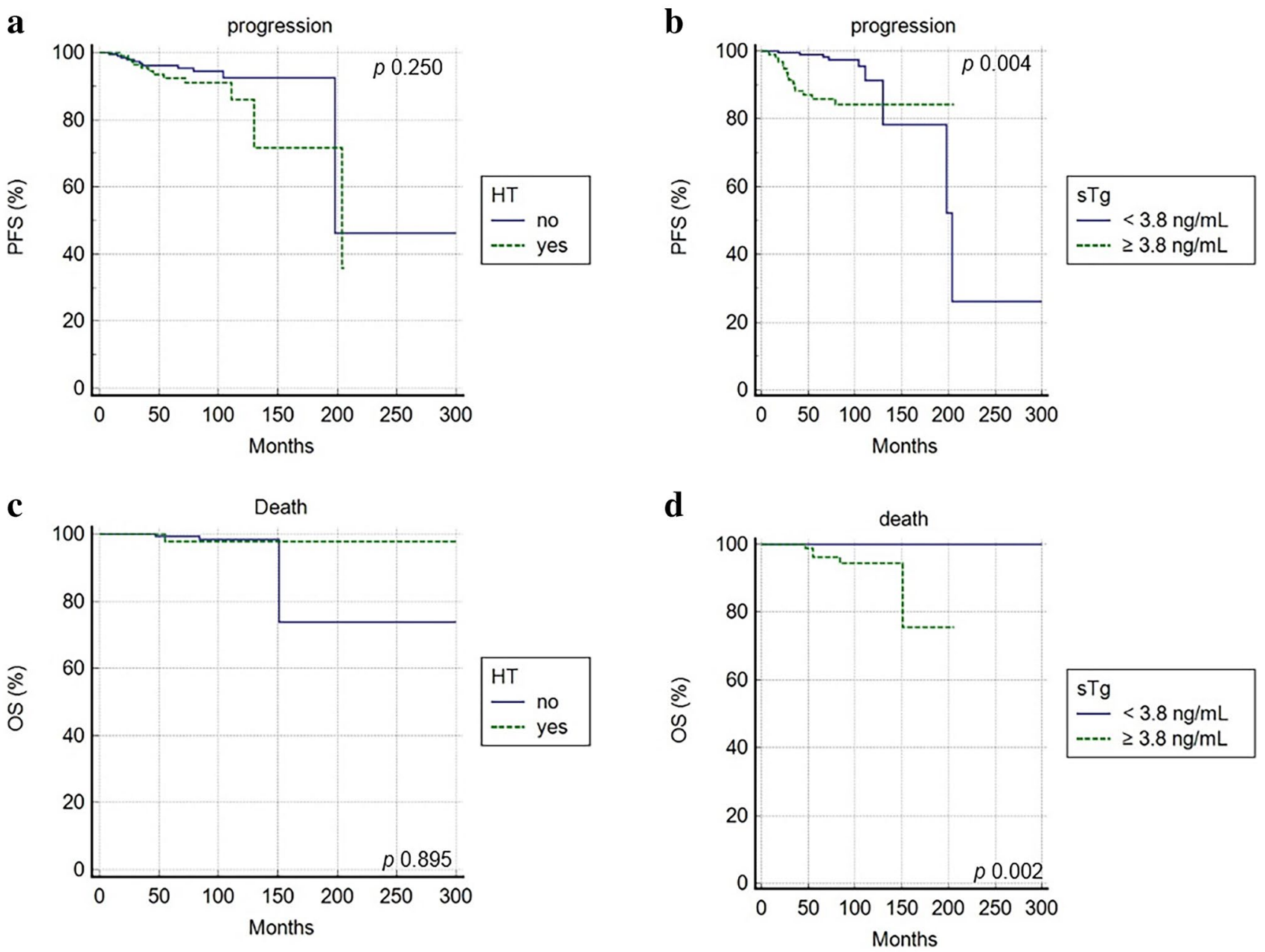

Fig. 3 Progression free survival a, b and overall survival c, d according to Hashimoto thyroiditis (HT) and stimulated thyroglobulin (sTg)

In conclusion, we have demonstrated that HT may affect the efficacy of RAIT in low- to intermediate-risk DTC, particularly reducing the successful rate of excellent response after RAIT. Instead, HT does not seem to have a prognostic role considering PFS and OS.

Funding Open access funding provided by Università degli Studi di Brescia within the CRUI-CARE Agreement.

\section{Declarations}

Conflict of interest The authors declare they have no conflict of interest.

Ethical approval All procedures performed in studies involving human participants were in accordance with the ethical standards of the institutional and/or national research committee and with the 1964 Helsinki declaration and its later amendments or comparable ethical standards. For this type of study formal consent is not required.

Informed consent Informed consent was obtained from all individual participant included in the study.

Open Access This article is licensed under a Creative Commons Attribution 4.0 International License, which permits use, sharing, adaptation, distribution and reproduction in any medium or format, as long as you give appropriate credit to the original author(s) and the source, provide a link to the Creative Commons licence, and indicate if changes were made. The images or other third party material in this article are included in the article's Creative Commons licence, unless indicated otherwise in a credit line to the material. If material is not included in the article's Creative Commons licence and your intended use is not permitted by statutory regulation or exceeds the permitted use, you will need to obtain permission directly from the copyright holder. To view a copy of this licence, visit http://creativecommons.org/licenses/by/4.0/. 


\section{References}

1. Sherman SI. Thyroid carcinoma. Lancet. 2003;361:501-11.

2. Haugen BR, Alexander EK, Bible KC, Doherty GM, Mandel SJ, Nikiforov YE, et al. 2015 American Thyroid Association Management Guidelines for adult patients with thyroid nodules and differentiated thyroid cancer: The American Thyroid Association Guidelines Task Force on thyroid nodules and differentiated thyroid cancer. Thyroid. 2016;26:1-133.

3. Albano D, Bertagna F, Bonacina M, Durmo R, Cerudelli E, Gazzilli M, et al. Possible delayed diagnosis and treatment of metastatic differentiated thyroid cancer by adopting the 2015 ATA guidelines. Eur J Endo. 2018;179:143-51.

4. Albano D, Panarotto MB, Durmo R, Rodella C, Bertagna F, Giubbini R. Clinical and prognostic role of detection timing of distant metastases in patients with differentiated thyroid cancer. Endocrine. 2019;63:79-86.

5. Tuttle RM, Ahuja S, Avram AM, Bernet VJ, Bourget P, Daniels $\mathrm{GH}$, et al. Controversies, consensus, and collaboration in the use of 131I therapy in differentiated thyroid Cancer: a joint statement from the American Thyroid Association, the European Association of Nuclear Medicine, the Society of Nuclear Medicine and Molecular Imaging, and the European Thyroid Association. Thyroid. 2019;29(4):461-70.

6. Jonklaas J, Cooper DS, Ain KB, Bigos T, Brierley JD, Haugen $\mathrm{BR}$, et al. Radioiodine therapy in patients with stage I differentiated thyroid cancer. Thyroid. 2010;20:1423-4.

7. Hindie E, Taieb D, Avram AM, Giovanella L. Radioactive iodine ablation in low-risk thyroid cancer. Lancet Diabetes Endocrinol. 2018;6:686.

8. Moten AS, Zhao H, Willis AI. The overuse of radioactive iodine in low-risk papillary thyroid cancer patients. Surg Oncol. 2019;29:184-9.

9. Albano D, Bonacina M, Durmo R, Bertagna F, Giubbini R. Efficacy of low radioiodine activity versus intermediate-high activity in the ablation of low risk differentiate thyroid cancer. Endocrine. 2020;68:124-31.

10. Schlumberger M, Catargi B, Borget I, Deandreis D, Zerdoud S, Bridji B, et al. Strategies of radioiodine ablation in patients with low-risk thyroid cancer. N Engl J Med. 2012;366:1663-73.

11. Mallick U, Harmer C, Yap B, Wadsley J, Clarke S, Moss L, et al. Ablation with low-dose radioiodine and thyrotropin alfa in thyroid cancer. N Engl J Med. 2012;366:1674-85.

12. Bai JB, Shakerian R, Westcott JD, Lichtenstein M, Miller JA. Factors influencing radioiodine uptake after thyroid cancer surgery. ANZ J Surg. 2013;85:572-7.

13. Bernier MO, Morel O, Rodien P, Muratet JP, Giraud P, Rohmer $\mathrm{V}$, et al. Prognostic value of an increase in the serum thyroglobulin level at the time of the first ablative radioiodine treatment in patients with differentiated thyroid cancer. Eur J Nucl Med Mol Imaging. 2005;32:1418-21.

14. Hackshaw A, Harmer C, Mallick U, Haq M, Franklyn JA. 131I activity for remnant ablation in patients with differentiated thyroid cancer: a systematic review. J Clin Endocrinol Metab. 2007;92:28-38.

15. Kwon H, Choi JY, Moon JH, Park HJ, Lee WW, Lee KE. Effect of Hashimoto thyroiditis on low-dose radioactive-iodine remnant ablation. Head Neck. 2015;38:E730-5.

16. Del Rio P, Montana C, Cozzani F, Rossini M, Loderer T, Dall'Aglio E, et al. Is there a correlation between thyroiditis and thyroid cancer? Endocrine. 2019;66:538-41.

17. Zhang Y, Dai J, Wu T, Yang N, Yin Z. The study of the coexistence of Hashimoto's thyroiditis with papillary thyroid carinoma. J Cancer Res Clin Oncol. 2014;140:1021-6.
18. Graceffa G, Patrone R, Vieni S, Campanella S, Calamia S, Laise I, et al. Association between Hashimoto's thyroiditis and papillary thyroid carcinoma: a retrospective analysis of 305 patients. BMC Endocr Disord. 2019;19:26.

19. Nagayama Y. Thyroid autoimmunity and thyroid cancer-the pathogenic conenction: a 2018 update. Horm Metab Res. 2018;50:922-31.

20. Noureldine SI, Tufano RP. Assoication of Hashimoro's thyroiditis and thyroid cancer. Curr Opin Oncol. 2015;27:21-5.

21. Pilli T, Toti R, Occhini R, Cantara S, Caselli M, Cardinale S, et al. Chronic lymphocytic thyroiditis (CLT) has a positive prognostic value in papillary thyroid cancer (PTC) patients: the potential key role of Foxp3+ T lymphocytes. J Endocrinol Invest. 2018;41:703-9.

22. Marotta V, Sciammarella C, Chiofalo MG, Gambardella C, Bellevicine C, Grasso M, et al. Hashimoto's thyroiditis predicts outcome in intrathyroidal papillary thyroid cancer. Endocr Relat Cancer. 2017;24:485-93.

23. Tuttle M, Morris LF, Haugen B, Shah J, Sosa JA, Rohren E, Subramaniam RM, Hunt JL, Perrier ND. Thyroid-differentiated and anaplastic carcinoma (Chapter 73). In: Amin MB, Edge SB, Greene F, Byrd D, Brookland RK, Washington MK, Gershenwald JE, Compton CC, Hess KR, Sullivan DC, Jessup JM, Brierley J, Gaspar LE, Schilsky RL, Balch CM, Winchester DP, Asare EA, Madera M, Gress DM, Meyer LR, editors. AJCC cancer staging manual. 8th ed. New York: Springer International Publishing; 2017.

24. Ragusa F, Fallahi P, Elia G, Gonnella D, Paparo SR, Giusti C, et al. Hashimto's thyroiditis: epidemiology, pathogenesis, clinic and therapy. Best Pract Res Clin Endocrinol Metabol. 2019;33:101367.

25. Derringer GA, Thompson LD, Frommelt RA, Bijwaard KE, Heffess CS, Abbondanzo SL. Malignant lymphoma of the thyroid gland: a clinicopathologic study of 108 cases. Am J Surg Pathol. 2000;24:623-39.

26. Kossev P, Livolsi V. Lymphoid lesions of the thyroid: review in light of the revised European-American lymphoma classification and upcoming World Health Organization classification. Thyroid. 1999;9:1273-80.

27. Dailey ME, Lindsay S, Skahen R. Relation of thyroid neoplasms to Hashimoto disease of the thyroid gland. AMA Arch Surg. 1955;70:291-7.

28. Lee JH, Kim Y, Choi JW, Kim YS. The association between papillary thyroid carcinoma and histologically proven Hashimoto's thyroiditis: a meta-analysis. Eur J Endocrinol. 2013;168:343-9.

29. Jankovic B, Le KT, Hershman JM. Clinical review: Hashimoto's thyroiditis and papillary thyroid carcinoma: is there a correlation? J Clin Endocrinol Metab. 2013;98:474-82.

30. McLeod DS, Watters KF, Carpenter AD, Ladenson PW, Cooper DS, Ding EL. Thyrotropin and thyroid cancer diagnosis: a systematic review and dose-response meta-analysis. J Clin Endocrinol Metab. 2012;97:2682-92.

31. Yildirim-Poyraz N, Yazgan A, Ozdemir E, Gozalan A, Keskin M, Ersoy R, et al. Predictive role of nontumoral sodium iodide symporter activity and preoperative thyroid characteristics in remission process of thyroid cancer patients. Ann Nucl Med. 2014;28:623-31.

32. Kollecker I, von Wasielewski R, Langner C, Muller JA, Spitzweg C, Kreipe H, et al. Subcellular distribution of the sodium iodide symporter in benign and malignant thyroid tissues. Thyroid. 2012;22:529-35.

33. Wagieh SM, El-Refaei SM, Salem SS, Al-Shiekh EA, AlGhamdy HA, Al-Juhani NR. Impact of histopathology of nonneoplastic thyroid tissue on ablation outcome in patients with papillary thyroid cancer. Nucl Med Commun. 2011;32:597-604. 
34. Tuttle RM, Tala H, Shah J, Leboeuf R, Ghossein R, Gonen $\mathrm{M}$, et al. Estimating risk of recurrence in differentiated thyroid cancer after total thyroidectomy and radioactive iodine remnant ablation: using response to therapy variables to modify the initial risk estimates predicted by the new American Thyroid Association staging system. Thyroid. 2010;20:1341-9.

35. Vaisman F, Momesso D, Bulzico DA, Pessoa CHCN, Dias F, Corbo R, et al. Spontaneous remission in thyroid cancer patients after biochemical incomplete response to initial therapy. Clin Endocrinol (Oxf). 2012;77:132-8.

36. Loh KH, Greenspan FS, Dong F, Miller TR, Yeo PP. Influence of lymphocytic thyroiditis on the prognostic outcome of patients with papillary thyroid carcinoma. J Clin Endocr Metabolism. 1999;84:458-63.

37. Jeong JS, Kim HK, Lee CR, Park S, Park JH, Kang SW, et al. Coexistence of chronic lymphocytic thyroiditis with papillary thyroid carcinoma: clinical manifestation and prognostic outcome. J Korean Med Sci. 2012;27:883-9.

38. Rosario PW, Barroso AL, Rezende LL, Padrao EL, Fagundes TA, Reis JS, et al. Outcome of ablation of thyroid remnants with $100 \mathrm{mCi}(3.7 \mathrm{GBq})$ iodine-131 in patients with thyroid cancer. Ann Nucl Med. 2005;19:247-50.

39. Chang YW, Kim HS, Jung SP, Kim HY, Lee JB, Bae JW, et al. Pre-ablation stimulated thyroglobulin is a better predictor of recurrence in pathological N1a papillary thyroid carcinoma than the lymph node ratio. Int J Clin Oncol. 2016;21:862-8.

Publisher's Note Springer Nature remains neutral with regard to jurisdictional claims in published maps and institutional affiliations. 